\title{
SHEAR FORCE ANALYSIS OF AN OIL-DRIVEN GRINDING TOOL
}

\author{
RODION GROLL \& CHRISTOPH KÜHN \\ Center of Applied Space Technology and Microgravity, University of Bremen, Germany.
}

\begin{abstract}
The main goal of the present grinding concept GrindBall is to develop a manufacturing process to machine micro structures [1] on hard and brittle materials, using a spherical and shaftless grinding tool. Thereby the negative effect of cutting speed variation caused by fixed rotational axes of conventional grinding pins is reduced. Spherical tools require new technologies for contactless tool drive and positioning [2] that are able to be miniaturised to a compact machining module and fit in the workspace of small machine tools [3]. Therefore, a fluidic drive and an electromagnetic bearing system were chosen for the GrindBall module as practically contactless technologies for power transmission [4].

Keywords: fluid/solid interaction, grinding tools, interfacial modelling, shear forces, two-phase flow.
\end{abstract}

\section{INTRODUCTION}

In order to determine the ideal geometry of the propulsion concept of the GrindBall module, to facilitate the selection of an appropriate propulsion fluid and to optimise the grinding forces produced by the tool, several numerical studies were performed (Fig. 1). The single and multi-phase fluid simulations are conducted with the open source CFD package OpenFOAM using an incompressible multi-phase solver capable of computing stresses at solid surfaces. A base geometry is determined through preliminary investigations, which are

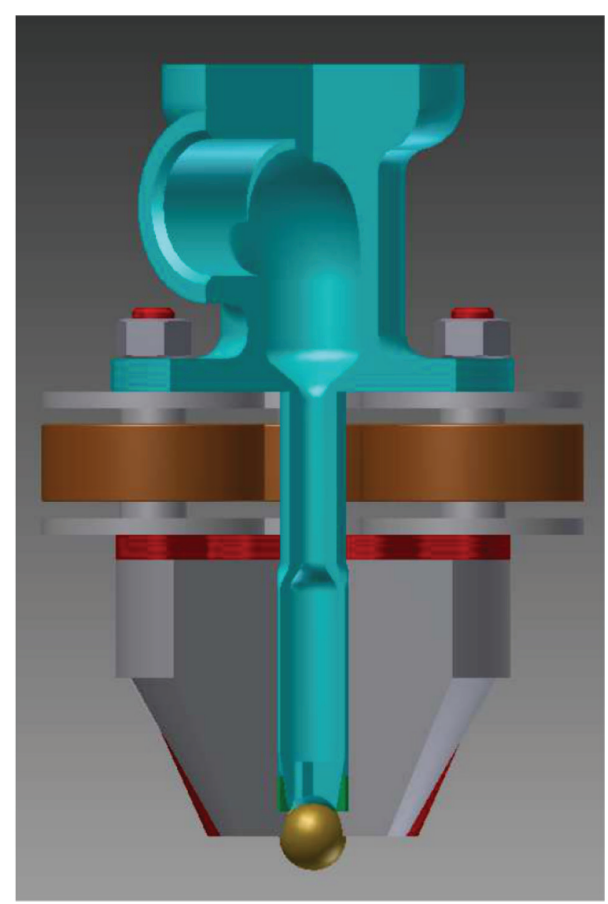

Figure 1: Grinding tool geometry with eccentric nozzle. 
based on practical and physical restrictions and on requirements for the grinding process [5]. The base geometry utilises a hydraulically powered spherical grinding tool with a diameter of $8 \mathrm{~mm}$. The force necessary to achieve abrasion is applied by the fluid flow, which is injected in a spherical gap through three vertical ducts (Fig. 2).

Although magnetic bearings are already successfully used in many technical systems, the design and control of magnetic bearings for a new, miniaturised module poses several technical challenges. For instance, it is particularly challenging to maintain a relatively small air gap between the spherical grinding tool, having a relatively low mass of approx. $2 \mathrm{~g}$, and a magnetic shaft [6]. The bearing concept must also consider the rotation of the tool, which induces eddy current effects. Hence, dimensioning and simulation of the system focuses on the optimisation of the magnetic bearing, electrodynamic effects and non-linear control [7]. The resulting shear force is analysed by the computational integration of the simulated shear stresses at the surface of the abrading sphere. The angular momentum is induced by single-phase flow and multi-phase flow (Fig. 3) through three collinear nozzles. The efficiency is discussed with an algebraic approach for the maximum of resulting forces in rotation direction.

\section{GOVERNING EQUATIONS}

The attachment point of the vertical flow onto the grinding body surface is shifted with the length $\delta$ from symmetry axis. The attaching forces work in flow direction with cosine ratio of the dynamic pressure depending on the relative velocity:

$$
\vec{F} d t=\Delta \vec{u} \cos \alpha \underbrace{d m}_{\dot{m} d t} .
$$

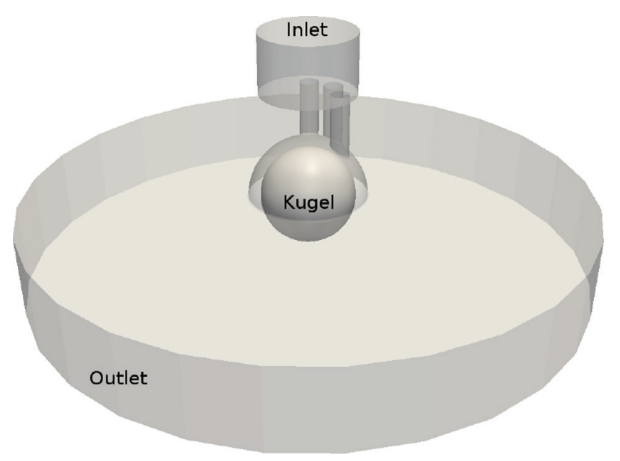

Figure 2: Geometry set-up with three eccentric nozzles into spherical gap.

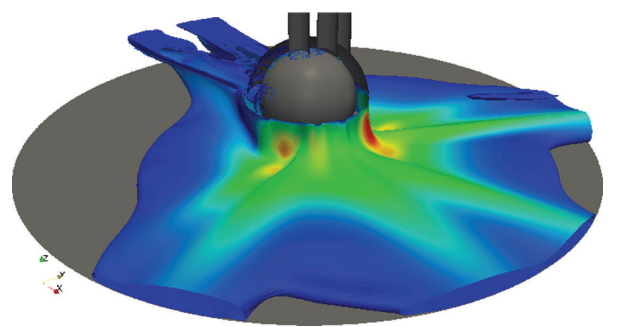

Figure 3: Surface level of outflowing liquid from tool in service. 
The force vector is derived by dividing eqn (1) by the time increment $d t$. The absolute force is described with the cosine ratio and the relative velocity of flow velocity and surface speed in angular direction:

$$
\begin{aligned}
|\vec{F}| & =(\dot{m} \Delta \vec{u} \cos \alpha) \cos \alpha \\
& =\dot{m}(U-R \Omega \sin \alpha) \underbrace{\cos ^{2} \alpha}_{\approx 1} \\
& \approx \dot{m}(U-R \Omega \sin \alpha) .
\end{aligned}
$$

This value describes the absolute force on the grinding body by the liquid flow stream.

\subsection{Angular momentum transfer}

The enforced angular momentum

$$
\vec{M}=\vec{r} \times \vec{F}
$$

is driven by the grinding sphere radius $R$ and the eccentric shift length $\delta$ :

$$
\begin{gathered}
R=|\vec{r}| \\
\delta=R \sin \alpha .
\end{gathered}
$$

Characteristic lengths and angles are sketched in Fig. 4. So the relation between absolute values is given as follows:

$$
|\vec{M}|=|\vec{F}| R \sin \alpha=|\vec{F}| \delta .
$$

With a general substitution of the nozzle velocity $U$ and the angular rotation speed $\Omega$ :

$$
\begin{gathered}
\Omega=2 \pi f \\
\dot{m}=\rho U A \Rightarrow U=\frac{\dot{m}}{\rho A}
\end{gathered}
$$

the absolute angular momentum value is written depending on rotation frequency $f$ and integrated nozzle square area $A$ as follows:

$$
\begin{aligned}
|\vec{M}| & =|\vec{M}| \delta \\
& =\dot{m} \delta(U-\Omega \delta) \\
& =\dot{m} \delta\left(\frac{\dot{m}}{\rho A}-2 \pi f \delta\right)
\end{aligned}
$$

The transferred angular momentum decreases with increasing rotation frequency. So positive momentum transfer can only be realized under a corresponding rotation frequency supremum.

\subsection{Friction efficiency}

Former investigations show a decreasing efficiency of the friction forces with higher rotation velocities of the grinding body. The friction efficiency $\eta$ is introduced for modelling this 


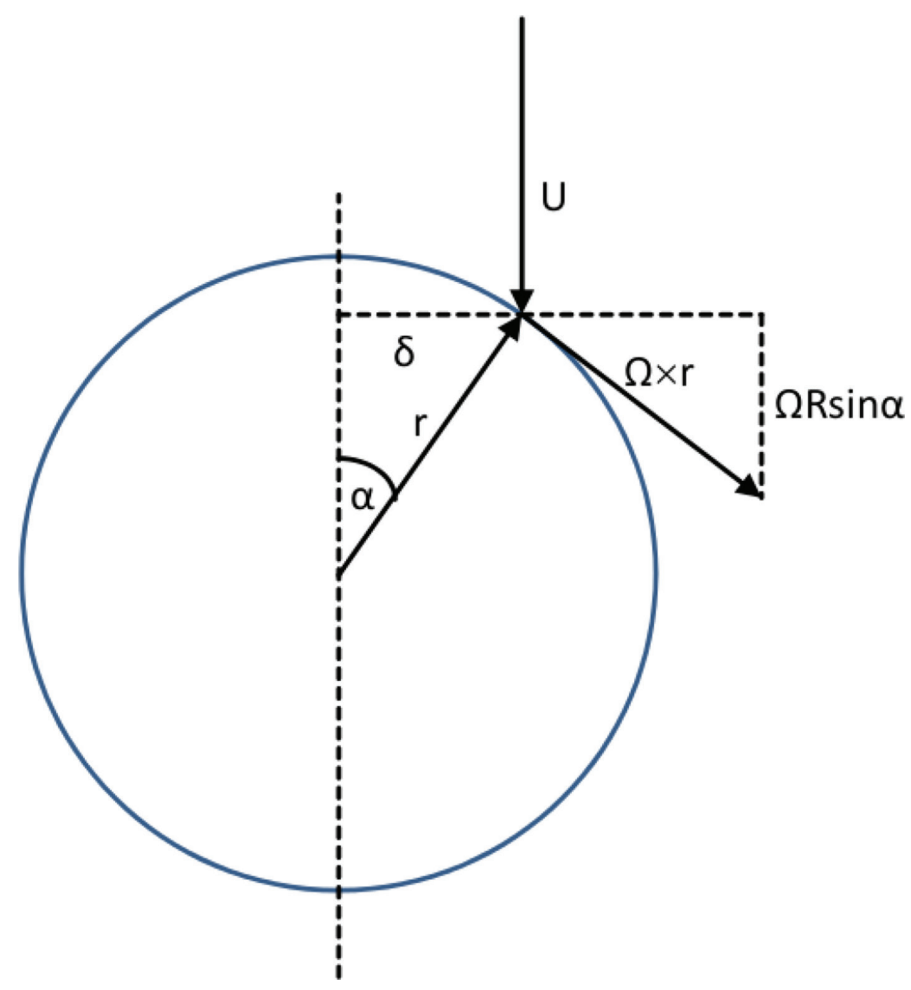

Figure 4: Attaching forces and angular momentums on spherical grinding body surface.

effect. The absolute shear force on the surface of the grinding sphere is corrected by this value:

$$
|\vec{F}|=\dot{m}\left(U-\frac{1}{\eta} R \Omega \sin \alpha\right) .
$$

The consequent substitution (eqn (7)) of the velocity values leads to the formulation of the angular momentum depending on friction efficiency:

$$
|\vec{M}|=\dot{m} \delta\left(\frac{\dot{m}}{\rho A}-2 \pi \frac{f}{\eta} \delta\right)
$$

The resulting grinding force $F_{G}$ is defined by the ratio of angular momentum and grinding sphere radius:

$$
\left|\vec{F}_{G}\right|=\frac{|\vec{M}|}{R}=\dot{m} \frac{\delta}{R}\left(\frac{\dot{m}}{\rho A}-2 \pi \frac{f}{\eta} \delta\right) .
$$

Hereby the relation between absolute grinding force, rotation speed and enforcing mass flow is developed. In Figs. 5-8 the plot of grinding forces $F_{G}[N]$ is shown in z-axis direction depending on rotation frequency $f[1 / \mathrm{s}]$ in x-axis direction and mass flow $\dot{m}[\mathrm{~kg} / \mathrm{s}]$ in y-axis direction. The effective grinding force (eqn (12)) is increasing with higher friction efficiency $\eta$. 


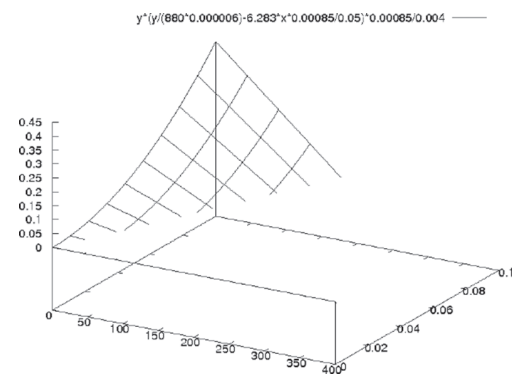

Figure 5: Grinding force with $\eta=5 \%$ efficiency.

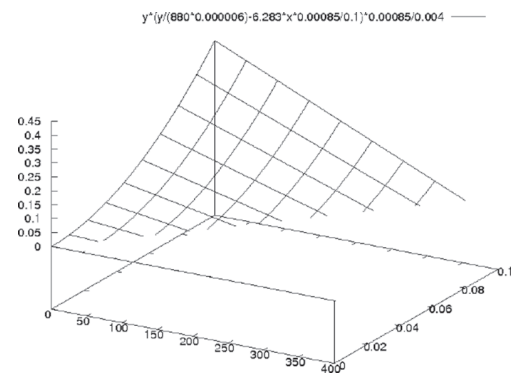

Figure 6: Grinding force with $\eta=10 \%$ efficiency.

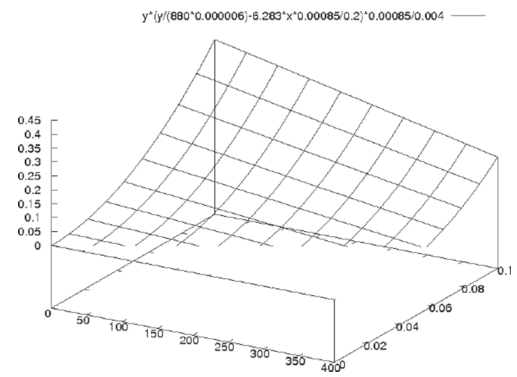

Figure 7: Grinding force with $\eta=20 \%$ efficiency.

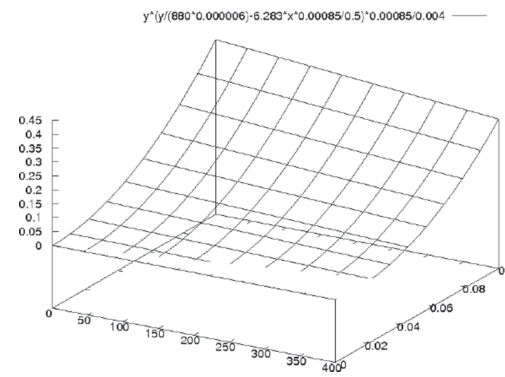

Figure 8: Grinding force with $\eta=50 \%$ efficiency. 


\section{MODELLING RESULTS}

The three-dimensional simulation data are approximated by modelling results of the present flow. Hereby a single-phase [8] and a multi-phase investigation (Figs 9 and 10) of the grinding process are compared with algebraic modelling approaches with estimated friction efficiencies. Geometric parameters are taken from the three-dimensional geometry. The integrated nozzle square-cut area has to be approximated by three circular areas with a diameter of $1.6 \mathrm{~mm}$ each:

$$
A=3 \frac{\pi}{4}(1.6 m m)^{2}=6.0 \cdot 10^{-6} \mathrm{~m}^{2}
$$

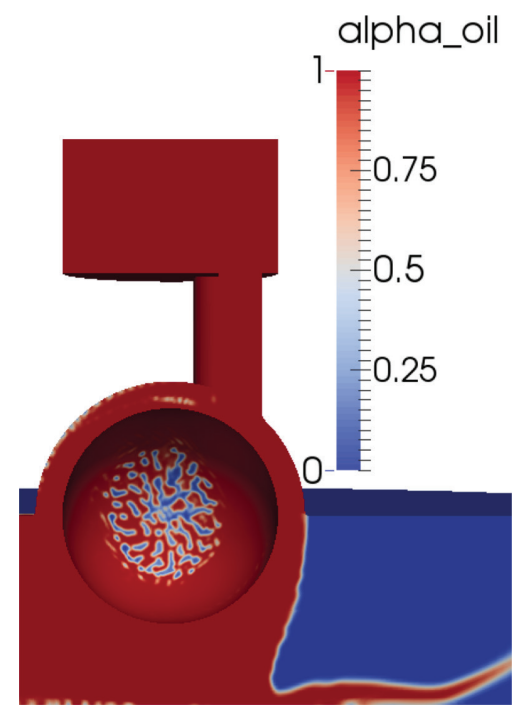

Figure 9: Two-phase grinding process with 3 1/min by $100 \mathrm{~Hz}$ rotation frequency.

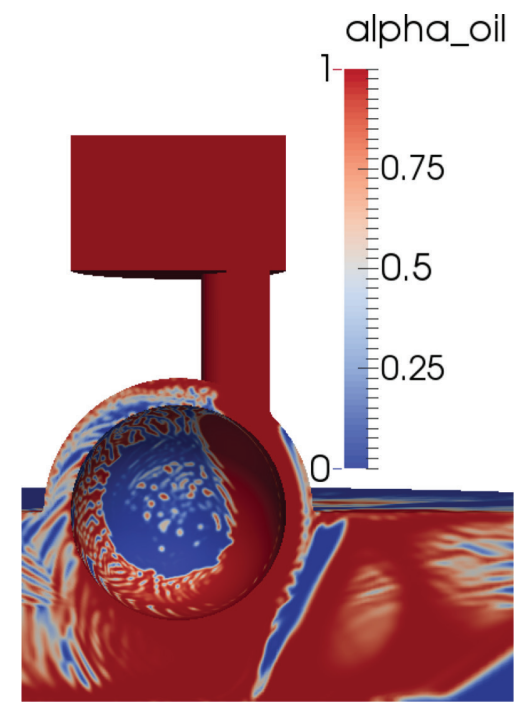

Figure 10: Two-phase grinding process with 5 1/min by $400 \mathrm{~Hz}$ rotation frequency. 


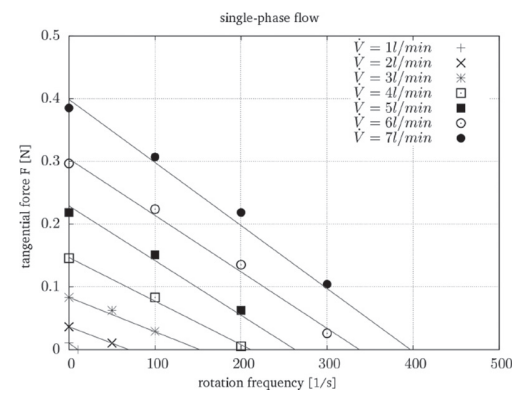

Figure 11: Grinding Forces of 3D single phase flow.

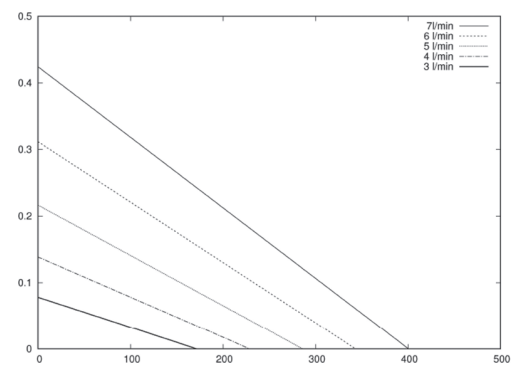

Figure 12: Modelled grinding forces with $11 \%$ efficiency.

The mean shift length is estimated with approximately

$$
\delta \approx 0.85 \mathrm{~mm} .
$$

At first a simple single-phase simulation of the present flow is investigated. The friction liquid is hydraulic oil HLP46 at a constant temperature of $293.15 \mathrm{~K}$ and atmospheric environmental pressure $101325 \mathrm{~Pa}$. The numerical results of a three-dimensional simulation are shown in Fig. 11. Approximated modelling curves (eqn (12)) are determinate in Fig. 12 with an estimated friction efficiency of $\eta=11 \%$. The second parameter study is a threedimensional simulation with a comparable volume-of-fluid code. The shear forces are investigated and also approximated by a linear regression curve (Fig. 13) like in the single-phase case. But the higher shear forces indicate a higher friction efficiency. The multi-phase results

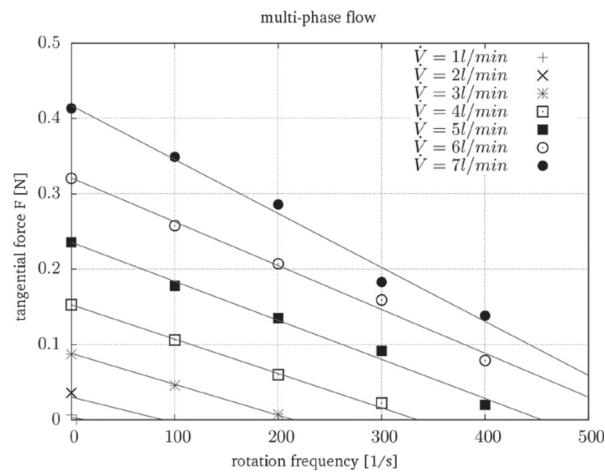

Figure 13: Grinding Forces of 3D multiphase flow. 


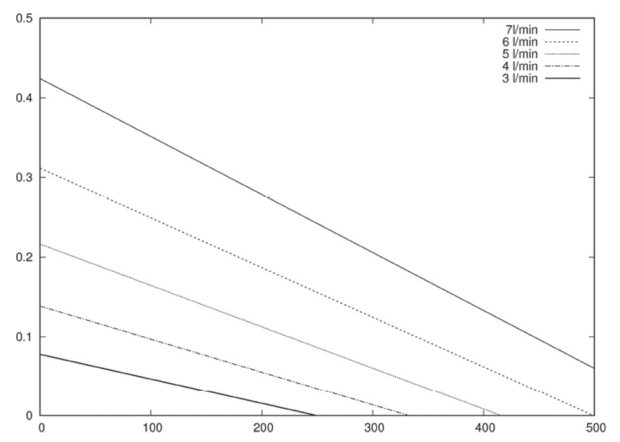

Figure 14: Modelled grinding forces with 16\% efficiency.

are approximated with a comparing modelling study of $\eta=16 \%$ (Fig. 14). The advantage of multi-phase flow usage can be explained by the missing of breaking liquid in the back of the grinding sphere. This liquid would be pumped into the gap between sphere and cavity wall in a single-phase flow. The resulting shear forces on the abrasive surface of the sphere would decrease transferred angular momentum. The missing of this breaking effect in the multi-phase set-up increases the transferred angular momentum by about $50 \%$ and the "friction efficiency" of the grinding tool from $11 \%$ to $16 \%$.

\section{PARAMETER DISCUSSION OF DIMENSIONLESS VALUES}

With a normalising transformation of the present formulation of the algebraic grinding force approximation (eqn (12))

$$
\frac{\left|\vec{F}_{G}\right|}{\underbrace{2 \pi f}_{\Omega} R \dot{m}\left(\frac{\delta}{R}\right)^{2}}=\frac{\dot{m}}{\underbrace{2 \pi f}_{\Omega} R^{3} \rho \frac{\delta}{R} \frac{A}{R^{2}}}-\frac{1}{\eta}
$$

the non-dimensional parameters of normalised grinding force $F_{G}^{*}$, normalised shift length $6^{*}$, normalised nozzle throat area $A^{*}$ and the friction efficiency $\eta$ :

$$
\begin{gathered}
F_{G}^{*}=\frac{\left|\vec{F}_{G}\right|}{\dot{m} R \Omega} \\
\dot{m}^{*}=\frac{\dot{m}}{\rho \Omega R^{3}} \\
\delta^{*}=\frac{\delta}{R} \\
A^{*}=\frac{A}{R^{2}}
\end{gathered}
$$

following relation results from the consequent substitution of the non-dimensional parameters:

$$
\frac{F_{G}^{*}}{\delta^{* 2}}=\frac{\dot{m}^{*}}{\delta^{*} A^{*}}-\frac{1}{\eta} .
$$


The grinding force is decreasing with increasing rotation frequency, as shown in Figs 11-14. For positive grinding forces the right side of eqn (20) also has to be positive. Therefore following relation is equal to the restriction of working region:

$$
\dot{m}^{*}>\frac{\delta^{*} A^{*}}{\eta} \Leftrightarrow \eta>\frac{\delta^{*} A^{*}}{\dot{m}^{*}} .
$$

This restriction also defines the needs of working tool layout.

\section{CONCLUSIONS}

The present approach shows good results for modelling hydraulic friction-depending grinding forces for single-phase and multi-phase processes. The advantage of the multi-phase set-up is shown by three-dimensional volume-of-fluid simulations. The new parameter of the friction efficiency $\eta$ characterises the difference of the behaviour.

With the present approach various families of hydraulic-driven working tools are able to be characterised. Restrictions of working range for mass flow and friction efficiency are shown with a dimensionless relation (eqn (21)).

\section{ACKNOWLEDGEMENTS}

The authors wish to thank the German Research Foundation (DFG) for providing funding for this project, the members of SPP1476 for their excellent cooperation and the North-German Supercomputing Alliance (HLRN) for granting access to their supercomputing facilities.

\section{REFERENCES}

[1] Dornfeld, D., Min, S. \& Takeuchi, Y., Recent advances in mechanical micro machining. CIRP Annals-Manufacuring Technology, 55(2), pp. 745-768, 2006. https://doi.org/10.1016/j.cirp.2006.10.006

[2] Denkena, B., Möhring, H. \& Kayapinar, H., Design of a compact fluidic xy-stage for precise positioning. Proceedings of the 7th International Conference on Micro Manufacturing - ICOMM2012, pp. 345-349, 2012.

[3] Wulfsberg, J., Redlich, T. \& Kohrs, P., Square foot manufacturing: a new production concept for micro manufacturing. Production Engineering, 4, pp. 75-83. https://doi.org/10.1007/s11740-009-0193-X

[4] Wulfsberg, J., Kohrs, P., Grimske, S. \& Röhlig, B., Square foot manufacturing: a new approach for desktop-sized reconfigurable machine tools. Future Trends in Production Engineering, pp. 201-208, 2013.

https://doi.org/10.1007/s11740-009-0193-x

[5] Leach, K., Groll, R. \& Rath, H., Large eddy simulation of a pneumatically powered abrading sphere. Fluid Flow Technology, 15, pp. 600-607, 2012.

[6] Brinksmeier, E., Orlik, B., Groll, R., Brandao, C., Norbach, A. \& Leach, K., Grindballmodul zum achsenlosen mikroschleifen. ANT Journal, 52(2), pp. 14-19, 2013.

[7] Brinksmeier, E., Orlik, B., Groll, R., Brandao, C., Norbach, A. \& Leach, K., Grindball - an innovative micro-grinding tool. Production Engineering, 7(5), pp. 469-476, 2013. https://doi.org/10.1007/s11740-013-0469-z

[8] Leach, K., Modelling Force Transfer in Boundary Layers of Moving Walls for Compressible and Incompressible Turbulent Flows Across Multiple Scales (ISBN: 9783954049103). Cuvillier, 2015. 